\title{
Health-Related Quality of Life of Patients with Hidradenitis Suppurativa Measured with the 15D Instrument and Comparison with the General Population and Patients with Psoriasis
}

\author{
Nicolas Kluger $^{a}$ Harri Sintonen $^{b}$ Martta Ranta $^{c}$ Martina Serlachius $^{c}$ \\ a University of Helsinki and Department of Dermatology and Allergology, Helsinki University Central Hospital, and \\ ${ }^{\mathrm{b}}$ Department of Public Health, University of Helsinki, Helsinki, and ${ }^{\mathrm{C}} \mathrm{AbbVie}$ Oy, Espoo, Finland
}

\section{Keywords}

Hidradenitis suppurativa $\cdot$ Health-related quality of life ·

$15 \mathrm{D}$ instrument $\cdot$ Finland

\begin{abstract}
Background: Hidradenitis suppurativa (HS) is a chronic inflammatory skin disease significantly impacting health-related quality of life (HRQoL). We measured the HRQoL of Finnish HS patients and compared it with that of the general population and psoriasis patients. Methods: Twenty-six diagnosed HS patients completed the 15D HRQoL questionnaire (15D), the Dermatology Life Quality Index (DLQI), and the Beck Depression Inventory-21 (BDI-21). The correlation between the results of these instruments was analyzed and the $15 \mathrm{D}$ results were compared with an age-standardized general population and psoriasis patients. Results: Compared with an age-standardized general population, HS patients scored statistically significantly worse on several 15D dimensions. A statistically significant difference compared to psoriasis patients was detected on the sexual activity dimension. Even though not statistically significant, the observed mean values of the remaining dimensions suggest that clinically important differences may exist. The 15D score
\end{abstract}

\section{KARGER}

() 2017 S. Karger AG, Basel

E-Mail karger@karger.com

www.karger.com/sad negatively correlated with the DLQI score $(r=-0.492 ; p=$ $0.011)$ and the BDI-21 score $(r=-0.592 ; p=0.001)$. Conclusions: $\mathrm{HS}$ is a serious condition, with an impact at least comparable to that of psoriasis. This study shows that the 15D is a feasible tool for examining HRQoL in HS patients.

(c) 2017 S. Karger AG, Basel

\section{Introduction}

Hidradenitis suppurativa (HS) is a chronic inflammatory skin disease of the terminal hair follicles, affecting the intertriginous skin area of axillary, genitofemoral, and perianal sites, which may lead to fistulae, draining wounds, and scarring [1]. HS has a more significant impact on health-related quality of life (HRQoL) [2-5] than other dermatologic conditions, including psoriasis, atopic dermatitis, and chronic urticaria [2]. To date, limited data exist on the HRQoL of Finnish HS patients. Using the 15D HRQoL questionnaire (15D) in a Finnish HS cohort $(n=26)$, we assessed HRQoL in a cross-sectional setting and compared these results with those of an agestandardized general population [6] and psoriasis patients [7]. 


\section{Materials and Methods}

\section{Study Population}

In 2015, we performed a single-center cross-sectional pilot study on the burden of HS in 26 patients. The study was conducted at the Department of Dermatology of the Helsinki University Central Hospital, Finland. The study enrolled adult patients diagnosed with HS. The inclusion criteria, study methodology, and main study results are reported in more detail elsewhere [8]. The patients' HRQoL was compared to that of a representative sample of an age-standardized general population $(n=4,176)$ from the Health 2011 Health Examination Survey [6] and to that of agestandardized psoriasis patients $(n=138)$ from the Health 2000 Health Examination Survey [7].

\section{Data Collection}

Data on demographics, social and economic status, history of HS, lifestyle, comorbidities, clinical presentation, and disease severity were collected from medical charts and during one study visit. Patients were asked to complete a set of questionnaires at home before the study visit, including the $15 \mathrm{D}$, the Dermatology Life Quality Index (DLQI), and the Beck Depression Inventory-21 (BDI-21).

The $15 D$

The $15 \mathrm{D}$ is a generic, multidimensional, standardized, self-administered HRQoL instrument with both profile and single index score properties [9]. It comprises 15 dimensions of health. Each dimension has 5 grades of severity; the patient must choose the level best describing his/her state of health at the moment (best level $=1$, worst level $=5$ ) [9]. The single index score (15D score), which represents overall HRQoL on a scale from 0 to 1 ( $1=$ full health, $0=$ dead), and the dimension level values (i.e., dimension scores), reflecting the goodness of the levels relative to no problems on the dimension $(=1)$ and to being dead $(=0)$, are calculated from the health state descriptive system (questionnaire) using a set of population-based preference or utility weights. A minimum clinically important change/difference in the $15 \mathrm{D}$ score has been estimated to be \pm 0.015 [10].

\section{Dermatology Life Quality Index}

The DLQI is the most frequently used instrument in randomized controlled trials in dermatology [11]. It is a simple, validated questionnaire that consists of 10 questions. Each question has 4 possible answers, for a maximum of 3 points, and a total maximum score of 30 . Higher scores indicate more severely affected HRQoL according to the following grade: no effect ( $0-1$ points), small effect ( $2-5$ points), moderate effect (6-10 points), very large effect (11-20 points), and extremely large effect (21-30 points).

\section{Beck Depression Inventory-21}

The BDI-21 is one of the most widely used instruments for measuring depression symptoms [12]. The questionnaire is composed of items relating to depressive, cognitive, and physical symptoms. The BDI- 21 consists of 21 questions and each question has 4 possible answers with a value of 0 to 3 assigned for each answer. Higher total scores indicate more severe depressive symptoms according to the following grade: normal ( $0-12$ points), mild depression (13-18 points), moderate depression (19-29 points), and severe depression ( $\geq 30$ points) [13].
Table 1. Demographics, clinical characteristics, comorbidities, and HRQoL scores in a cohort of Finnish patients with HS (total $n=$ 26)

$\begin{array}{ll}\text { Demographics } & \\ \text { Age, years } & 44.2 \pm 15.5 \\ \text { Age at onset, years } & 26.8 \pm 14.9 \\ \text { Age at diagnosis, years } & 40.5 \pm 15.0 \\ \text { Duration of HS, years } & 18.4 \pm 10.2 \\ \text { BMI } & 31.2 \pm 7.31\end{array}$

\begin{tabular}{ll}
\hline Lifestyle & \\
Smoking & $20 / 26(76.9 \%)$ \\
Alcohol consumption & $19 / 26(73.1 \%)$
\end{tabular}

$\begin{array}{lc}\text { Disease severity } & \\ \text { Hurley stage } & \\ \text { Stage I } & 13 / 26(50 \%) \\ \text { Stage II } & 11 / 26(42.3 \%) \\ \text { Stage III } & 2 / 26(7.7 \%) \\ \text { Number of affected areas }^{a} & 2.04 \pm 1.58\end{array}$

Comorbidities

Obesity $(\mathrm{BMI}>30)$

$13 / 26(50 \%)$

Diabetes type II

$4 / 26(15.4 \%)$

Cardiovascular diseases

$9 / 26(34.6 \%)$

Autoimmune or inflammatory diseases ${ }^{\mathrm{b}} \quad 5 / 26(19.2 \%)$

Depression/anxiety disorders

$4 / 26(15.4 \%)$

HRQoL scores

$15 \mathrm{D}$

$0.882 \pm 0.083$

DLQI

$8.31 \pm 7.39$

BDI-21

$10.69 \pm 10.13$

Values are presented as mean \pm SD or $n(\%)$. 15D, 15D healthrelated quality of life questionnaire; BDI-21, Beck Depression Inventory-21; DLQI, Dermatology Life Quality Index; HRQoL, health-related quality of life; HS, hidradenitis suppurativa; SD, standard deviation. ${ }^{\text {a }}$ Axillae, inguinal folds, buttocks, breast, and scrotum. ${ }^{b}$ Hypothyroidism, Crohn disease, autoimmune cholangitis, myasthenia, and cutaneous lupus.

\section{Statistical Analysis}

Statistical analysis was conducted using SPSS Statistics, version 22.0 (IBM, Armonk, NY, USA). Patient characteristics are presented as mean and standard deviation (SD) for continuous variables and as frequency and proportion for categorical variables. The statistical significance of the differences between genders in the means of continuous variables was tested using an independent-samples $t$ test. Correlations between patients' age, disease duration, number of affected areas, Hurley stage, comorbidities, smoking and alcohol consumption, DLQI, BDI-21, and total $15 \mathrm{D}$ score were analyzed using the Spearman rank correlation coefficient. $p<0.05$ was considered statistically significant. 


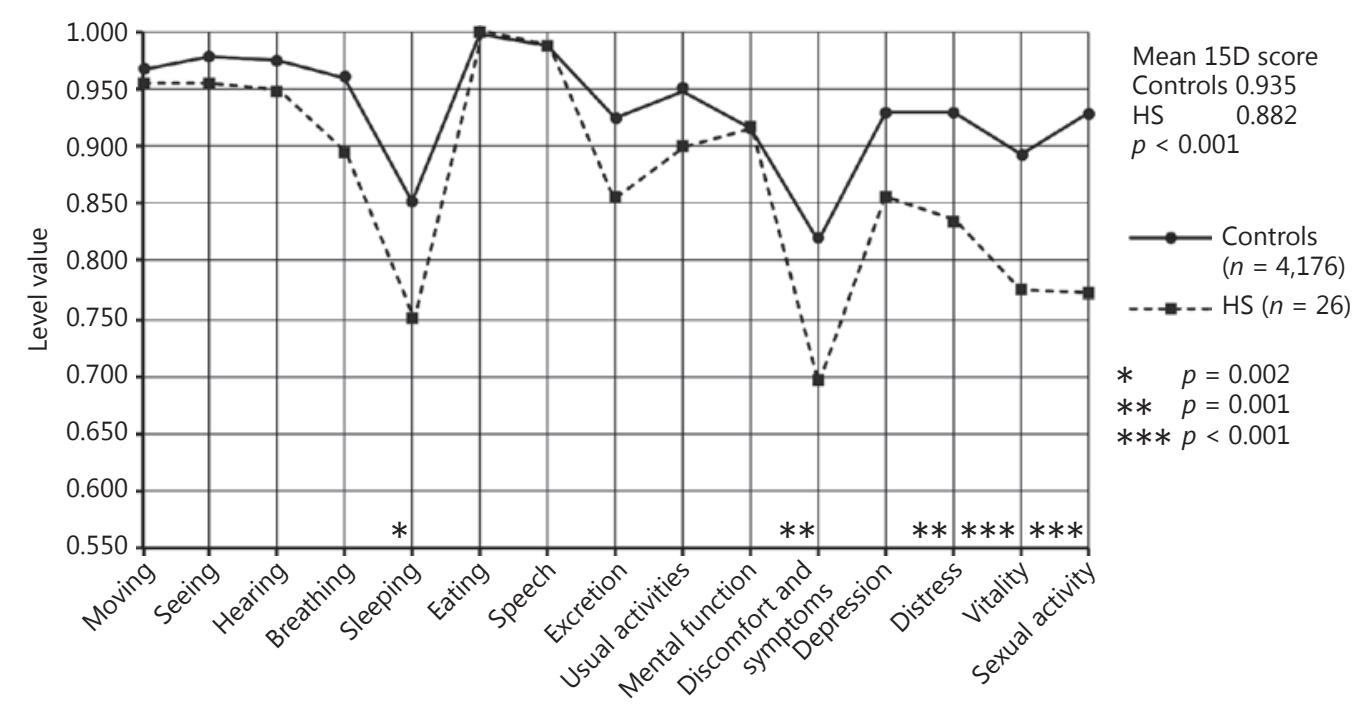

a

Dimensions

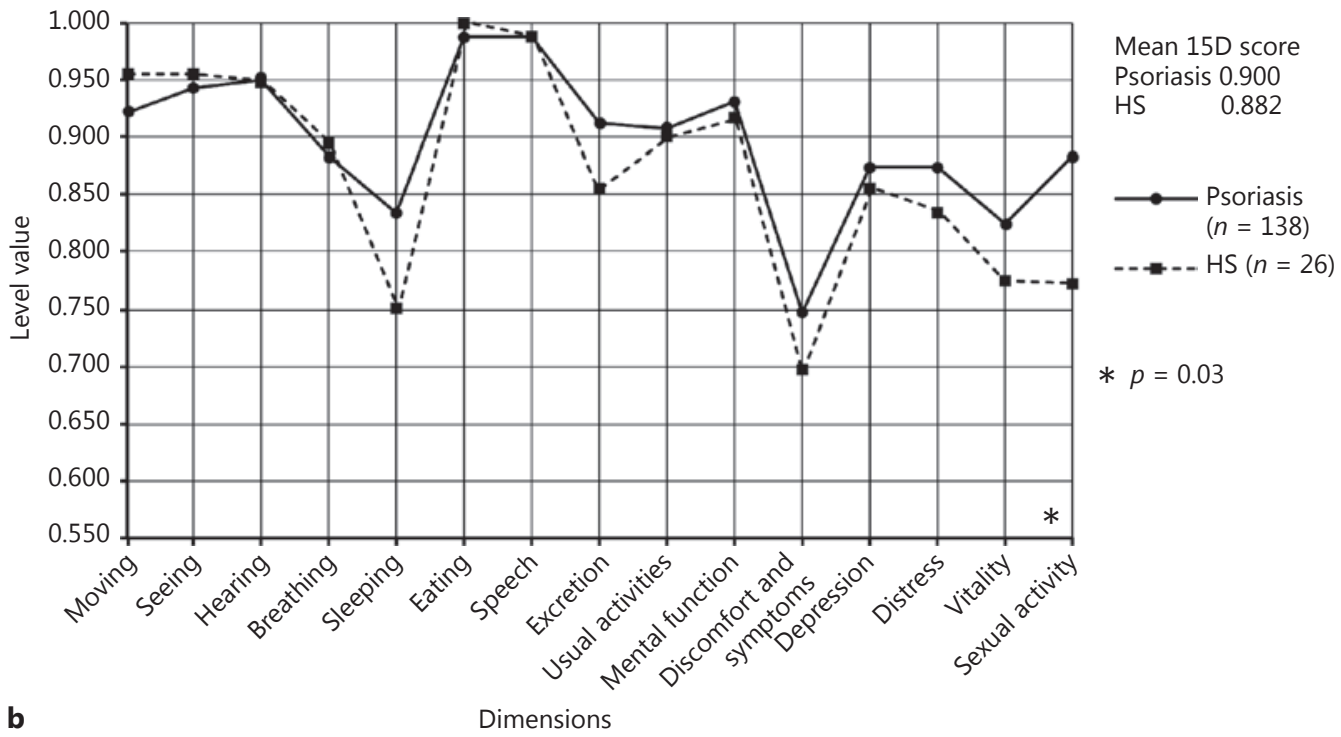

Fig. 1. a Mean 15D health-related quality of life questionnaire (15D) scores and profiles of patients with hidradenitis suppurativa (HS) $(n=26)$ and an age-standardized population control cohort $(n=4,176)$. b Mean $15 \mathrm{D}$ scores and profiles of patients with HS $(n=26)$ and an age-standardized population of patients with psoriasis $(n=138)$.

\section{Results}

\section{Demographic Data and HS Presentation}

The characteristics of the cohort, including demographic data, clinical characteristics, comorbidities, and HRQoL scores are summarized in Table 1.

\section{DLQI and BDI-21}

The mean (SD) DLQI score was $3.00 \pm 3.77$ for men and $11.63 \pm 7.22$ for women, suggesting statistically significantly lower HRQoL for women $(p=0.001)$. Nine patients $(34.6 \%)$, all women, reported a very large to extremely large effect on their daily life (DLQI $>10)$. The 
mean (SD) total BDI-21 score was statistically significantly worse for women compared to men $(15.00 \pm 0.078$ vs. $0.906 \pm 0.090$, respectively; $p=0.001$ ). Ten patients (38.5\%), of whom 9 were women, reported depression symptoms (BDI-21 >12); mild, moderate, and severe depression was reported in 6,2 , and 2 patients, respectively. The DLQI and BDI-21 results are discussed in detail elsewhere [8].

\section{Comparison of $15 D$ Results in HS Patients,}

an Age-Standardized General Population, and

Psoriasis Patients

There was no statistically significant difference in the mean (SD) 15D score between men and women, although the difference was clinically important $(0.906 \pm 0.090$ vs. $0.866 \pm 0.078$, respectively; $p=0.248$ ). HS patients had a statistically significantly lower mean (SD) 15D score compared with the age-standardized general population $(0.882 \pm 0.083$ vs. $0.935 \pm 0.071 ; p<0.001$; Fig. $1 \mathrm{a})$ and the difference was clinically important. In HS patients, 15D scores ranged from 0.668 to 1.000 . Only 1 patient (3.8\%) reported full health (i.e., a 15D score of 1). HS patients reported worse scores on all single dimensions, except for eating, speech, and mental function. The mean dimension scores of HS patients were statistically significantly worse compared to the general population in sleeping $(p=0.002)$, discomfort and symptoms $(p=0.001)$, distress $(p=0.001)$, vitality $(p<0.001)$, and sexual activity dimensions ( $p<0.001$; Fig. 1a).

HS patients had a lower mean (SD) 15D score compared with the age-standardized psoriasis population $(0.882 \pm 0.083$ vs. $0.900 \pm 0.117)$, but the difference was not statistically significant (independent-samples $t$ test, $p>0.05)$. The global pattern of the dimension scores was similar between both groups (Fig. 1b). However, HS patients on average scored worse on sleeping, excretion, discomfort and symptoms, distress, vitality, and sexual activity dimensions. The only statistically significant difference between patients with psoriasis and HS was on the dimension of sexual activity $(p=0.03)$.

Among HS patients, statistically significant correlations were not observed between the 15D score and age, disease duration, number of affected areas, Hurley stage, comorbidities, smoking, or alcohol consumption. However, the 15D score negatively correlated with the DLQI score $(r=-0.492 ; p=0.011)$ and the BDI-21 score $(r=$ $-0.592 ; p=0.001)$.

\section{Discussion}

As reported earlier, HS has a notable impact on HRQoL [2]. The age-standardized general population scored better on 12 of the 15 dimensions compared with the study group. HS patients scored particularly worse on the dimension of discomfort and symptoms. Indeed, HS lesions may be painful and associated with malodorous discharge, impacting everyday life and social relationships. Scores were also significantly lower on sleeping, usual activities, distress, depression, vitality, and sexual activity dimensions. Even though not statistically significant $(p=$ 0.082 ), excretion scored low. This dimension may be affected mainly in HS patients with inflammatory bowel disease or perianal fistulas and in patients with HS lesions on the buttocks and groin areas.

When comparing $15 \mathrm{D}$ results between HS and psoriasis patients, both patient groups interestingly displayed a similar pattern of impairment. HS patients had a lower mean 15D score than psoriasis patients, but unlike Wolkenstein et al. [2], we were unable to detect a statistically significant difference in terms of the global impact on patients' HRQoL. Differences in the sample size, population selection, and HRQoL questionnaires are most likely responsible for the discrepancies. In our cohort, HS patients showed a statistically significant impairment in sexual activity compared to psoriasis patients. Kurek et al. [4] previously reported that HS patients display higher sexual dysfunction scores, even when compared to psoriasis patients with genital involvement or underlying depression.

Due to the small sample size we did not have enough statistical power to detect small differences. It is noteworthy to mention that psoriasis patients were not examined and the severity of psoriasis could not be assessed. Acknowledging the limitations of our study, our results are in agreement with previous publications regarding the impact of HS on HRQoL. Importantly, our study shows that the $15 \mathrm{D}$ is a feasible and easily applied tool for examining HRQoL in HS patients. Scores from the 15D showed concordance with both DLQI and BDI-21 scores. In the near future, we aim at extending this study on a nationwide scale.

\section{Acknowledgments}

The authors wish to thank Juha Turunen, $\mathrm{PhD}$, of Farenta Oy for data management services, and Teppo Huttunen of 4 Pharma for conducting the statistical analyses. The authors also wish to thank Jenna Olkkonen, RN, for her assistance, and the patients for their cooperation and willingness to participate in our study. Brian Calimlim, DRPH, MS, of AbbVie is acknowledged for his valuable 
input during the review of the manuscript. The design, study conduct, and financial support for the study were provided by AbbVie. AbbVie participated in the interpretation of data, review, and approval of the publication.

\section{Statement of Ethics}

All procedures performed in this study were in accordance with the Helsinki Declaration of 1975, as revised in 1983. The Ethics Committee of HUCH Internal Medicine approved the study $(191 / 13 / 03 / 01 / 15)$. Informed consent was obtained from all study subjects.

\section{Disclosure Statement}

Dr. Nicolas Kluger has served as a consultant to AbbVie and has received research funding from AbbVie and speaker fees from AbbVie and Galderma. Harri Sintonen is the developer of the 15D and obtains royalties from its electronic versions. Martta Ranta and Martina Serlachius are employees of AbbVie. The sponsor of this study, AbbVie, participated in the study design, study research, collection, analysis and interpretation of data, and writing, reviewing, and approval of this publication. All authors had access to the data and participated in the development, review and approval, and decision to submit this publication.

\section{References}

1 Zouboulis CC, Desai N, Emtestam L, Hunger RE, Ioannides D, Juhász I, Lapins J, Matusiak L, Prens EP, Revuz J, Schneider-Burrus S, Szepietowski JC, van der Zee HH, Jemec GB: European S1 guideline for the treatment of hidradenitis suppurativa/acne inversa. J Eur Acad Dermatol Venereol 2015;29:619-644.

2 Wolkenstein P, Loundou A, Barrau K, Auquier P, Revuz J; Quality of Life Group of the French Society of Dermatology: Quality of life impairment in hidradenitis suppurativa: a study of 61 cases. J Am Acad Dermatol 2007; 56:621-623.

3 Matusiak E, Bieniek A, Szepietowski JC: Hidradenitis suppurativa markedly decreases quality of life and professional activity. J Am Acad Dermatol 2010;62:706-708.

4 Kurek A, Peters EM, Chanwangpong A, Sabat R, Sterry W, Schneider-Burrus S: Profound disturbances of sexual health in patients with acne inversa. J Am Acad Dermatol 2012;67: 422-428.
5 Esmann S, Jemec GB: Psychosocial impact of hidradenitis suppurativa: a qualitative study. Acta Derm Venereol 2011;91:328-332.

6 Koskinen S, Lundqvist A, Ristiluoma N: Health, Functional Capacity and Welfare in Finland in 2011. National Institute for Health and Welfare (THL), Report 68/2012. https:// www.julkari.fi/bitstream/handle/10024/ 90832/Rap068_2012_netti.pdf (accessed January 12,2017$)$.

7 Aromaa A, Koskinen S: Health and Functional Capacity in Finland. Baseline Results of the Health 2000 Health Examination Survey. Helsinki, Publications of the National Public Health Institute B12, 2004. http://thl32-kk. lib.helsinki.fi/bitstream/handle/ 10024/78534/KTLB12-2004.pdf? sequence=1 (accessed January 12, 2017).

8 Kluger N, Ranta M, Serlachius M: The burden of hidradenitis suppurativa in a cohort of patients in southern Finland: a pilot study. Skin Appendage Disord 2017;3:20-27.
9 Sintonen H: The 15D instrument of healthrelated quality of life: properties and applications. Ann Med 2001;33:328-336.

10 Alanne S, Roine RP, Räsänen P, Vainiola T, Sintonen H: Estimating the minimum important change in the 15D scores. Qual Life Res 2015;24:599-606.

11 Finlay AY, Khan GK: Dermatology Life Quality Index (DLQI) - a simple practical measure for routine clinical use. Clin Exp Dermatol 1994;19:210-216.

12 Beck AT, Ward CH, Mendelson M, Mock J, Erbaugh J: An inventory for measuring depression. Arch Gen Psychiatry 1961;4:561571.

13 Beck AT: Measuring depression: the depression inventory; in Williams TA, Katz MM, Shield JA (eds): Recent Advances in the Psychobiology of the Depressive Illnesses. Washington, DC, Government Printing Office, 1972, pp 299-302. 